\title{
Melatonin and Human Cardiovascular Disease
}

Journal of Cardiovascular Pharmacology and Therapeutics 2017, Vol. 22(2) 122-132

(C) The Author(s) 2016

Reprints and permission: sagepub.com/journalsPermissions.nav DOI: I0.1 I77/I0742484|6660622 journals.sagepub.com/home/cpt

(A)AGE

\section{Seithikurippu R. Pandi-Perumal, MSc', Ahmed S. BaHammam, MD, FACP', Nwakile I. Ojike, MD, $\mathbf{M S}^{2}$, Oluwaseun A. Akinseye, MD $^{3,4}$, Tetyana Kendzerska, MD, PhD $^{5}$, Kenneth Buttoo, MD, FRCP(C) ${ }^{6}$, Perundurai S. Dhandapany, MSc, PhD ${ }^{7,8,9,10}$, Gregory M. Brown, MD, PhD ${ }^{11}$, and Daniel P. Cardinali, MD, PhD ${ }^{12}$}

\begin{abstract}
The possible therapeutic role of melatonin in the pathophysiology of coronary artery disorder (CAD) is increasingly being recognized. In humans, exogenous melatonin has been shown to decrease nocturnal hypertension, improve systolic and diastolic blood pressure, reduce the pulsatility index in the internal carotid artery, decrease platelet aggregation, and reduce serum catecholamine levels. Low circulating levels of melatonin are reported in individuals with CAD, arterial hypertension, and congestive heart failure. This review assesses current literature on the cardiovascular effects of melatonin in humans. It can be concluded that melatonin deserves to be considered in clinical trials evaluating novel therapeutic interventions for cardiovascular disorders.
\end{abstract}

\section{Keywords}

blood pressure, cardioprotection, cardiovascular disorders, coronary artery disease, hypertension, platelets, melatonin

\section{Background}

Cardiovascular diseases (CVD) are the leading cause of death globally, with estimated 17.5 million deaths in 2012 , representing $31 \%$ of all global deaths. ${ }^{1}$ In the United States, about 1 in every 4 deaths is attributed to CVD with a direct cost of US\$312.6 billion in the year $2011 .^{2}$ Although there is a documented improvement in mortality rate from CVD, the overall impact such as survival with disability, dependency, and cost of care has significantly increased in the past decades. ${ }^{3}$ There has equally been an extensive improvement in the knowledge and understanding of the pathophysiology of CVDs over the last years with advances in pharmacological and procedural interventions.

\footnotetext{
'Department of Medicine, College of Medicine, The University Sleep Disorders Center, King Saud University, Riyadh, Saudi Arabia

${ }^{2}$ Division of Health and Behavior, Department of Population Health, New York University Medical Center, Center for Healthful Behavior Change, New York, NY, USA

${ }^{3}$ Department of Internal Medicine, Icahn School of Medicine at Mount Sinai, Queens Hospital Center, New York, NY, USA

${ }^{4}$ CUNY School of Public Health at Brooklyn College, New York, NY, USA

${ }^{5}$ Institute for Clinical Evaluative Sciences, Sunnybrook Health Sciences Center, Toronto, Ontario, Canada

${ }^{6}$ Sleep Disorders Center, Ajax, Ontario, Canada

${ }^{7}$ The Knight Cardiovascular Institute, Oregon Health and Science University, Portland, OR, USA

${ }^{8}$ Department of Medicine, Oregon Health and Science University, Portland, OR, USA

${ }^{9}$ Department of Molecular and Medical Genetics, Oregon Health and Science University, Portland, OR, USA

${ }^{10}$ Centre for Cardiovascular Biology and Disease, Institute for Stem Cell Biology and Regenerative Medicine (inStem), Bangalore, India

1 Centre for Addiction and Mental Health, University of Toronto, Toronto, Ontario, Canada

12 BIOMED-UCA-CONICET and Department of Teaching and Research, Faculty of Medical Sciences, Pontificia Universidad Católica Argentina, Buenos Aires, Argentina
}

Manuscript submitted: February 25, 2016; accepted: May 3I, 2016.

\section{Corresponding Authors:}

Ahmed S. BaHammam, University Sleep Disorders Centre, College of Medicine, King Saud University, Box 225503, Riyadh II 324, Saudi Arabia.

Email: ashammam2@gmail.com

Daniel P. Cardinali, BIOMED-UCA-CONICET and Department of Teaching and Research, Faculty of Medical Sciences, Pontificia Universidad Católica Argentina, 1107 Buenos Aires, Argentina.

Email: danielcardinali@fibertel.com.ar 
The focus of this review article is on the therapeutic potential of melatonin in CVDs. Melatonin (IUPAC name: N-[2-(5methoxy-1H-indol-3-yl) ethyl] acetamide) is a natural methoxyindole first described as a pineal hormone and later shown to bepresent in most mammalian and nonmammalian cells. ${ }^{4}$ Its effect is thought to be mediated through both receptormediated and receptor-independent mechanisms. The receptor-mediated actions of melatonin comprise of membrane melatonergic receptors (MT1 and MT2) located throughout the vascular system including the heart (cardiomyocytes, left ventricle, and coronary arteries). ${ }^{5,6}$ Melatonin may also be the natural ligand for the retinoid-related orphan nuclear hormone receptor family (RZR/ROR). ${ }^{7}$

The MT1 melatonergic receptors mediate arterial vasoconstriction, inhibit neuronal firing and cell proliferation in cancer cells, and modulate reproductive and metabolic functions. ${ }^{8,9}$ Activation of MT2 melatonergic induces vasodilation, phase shift circadian rhythms of neuronal firing in the suprachiasmatic nucleus, enhances immune responses, and inhibits dopamine release in retina and leukocyte rolling in arterial beds. The receptor-independent mechanism of action of melatonin is achieved through its antioxidant and mitochondrial-protecting effects. ${ }^{7}$

Melatonin has been shown to decrease nocturnal hypertension, ${ }^{10}$ reduce the pulsatility index in the internal carotid artery, decrease platelet aggregation, ${ }^{11,12}$ and reduce serum catecholamine levels. ${ }^{13}$ Moreover, decreased melatonin levels were reported in various pathological conditions including hypertension with nondipper pattern, ${ }^{14}$ congestive heart failure (CHF) ${ }^{15}$ ischemic heart disease, ${ }^{16}$ or in patients after acute myocardial infarction. ${ }^{17}$ Figure 1 presents the functional pleiotropy of melatonin.

This article provides a review of current literature on the cardiovascular effects of melatonin in humans. Medical literature was identified by searching databases including (MEDLINE, EMBASE), bibliographies from published literature, and clinical trial registries/databases. Searches were last updated on August 10, 2015.

\section{Basic Aspects of Melatonin Physiology Relevant to Cardiovascular Physiopathology}

By using specific melatonin antibodies, the presence of melatonin has been verified in multiple extrapineal tissues such as the brain, retina, lens, cochlea, Harderian gland, airway epithelium, gastrointestinal tract, liver, kidney, thyroid, pancreas, thymus, spleen, immune system cells, skin, carotid body, reproductive tract, and endothelial cells. ${ }^{18}$ For further details, the reader is referred to a review by Acuña-Castroviejo et al. ${ }^{18}$ Whether melatonin is produced in those tissues is a matter of debate because melatonin's amphiphilicity would allow an easy entry from circulation in most cases. ${ }^{19}$ However, in some tissues, melatonin concentrations exceed those in the blood. ${ }^{20}$ Although the enzymatic machinery to produce melatonin is found in most of these locations, ${ }^{18}$ circulating melatonin in mammals is derived exclusively from the pineal gland.
Melatonin effects on the vasculature depend on the specific receptor type activated. Animal studies reveal that vasoconstriction is mediated through MT1 activation and vasorelaxation through MT2 activation, with the likely mechanism of action being via the modulation of the noradrenergic and/or nitric oxide $(\mathrm{NO})$ effect. $^{21}$

Melatonin is metabolized in the liver to 6-sulfatoxymelatonin (aMT6s), which is subsequently excreted in urine. ${ }^{22}$ Melatonin that is produced outside the pineal gland generally does not reach the circulation, for example, in case of the gastrointestinal tract, melatonin goes through a high presystemic hepatic elimination rate and therefore does not exert systemic effects. $^{23}$

Reactive oxygen and nitrogen species are significant contributors to cardiac damage during ischemia-reperfusion injury after an acute coronary syndrome. The reported lower serum level of melatonin in this group of individuals (Table 1) worsens the possibility of further cardiac damage from ischemiareperfusion injury because melatonin has been described as a direct free radical scavenger that protects against reactive oxygen and nitrogen species with high efficacy. ${ }^{30}$ Melatonin also indirectly stimulates antioxidative enzymes such as superoxide dismutase, glutathione peroxidase, glutathione reductase, and glucose-6-phosphate dehydrogenase, thereby lowering molecular damage under conditions of elevated oxidative stress such as acute coronary syndrome. ${ }^{30}$

Because of its highly lipophilic properties, melatonin crosses all cell membranes and easily reaches subcellular compartments, including mitochondria and nuclei, where it may accumulate in high amounts. ${ }^{18,31}$ Melatonin counteracts lipid peroxidation $^{32}$ and DNA damage. ${ }^{33}$ In particular, melatonin preserves normal mitochondrial function by reducing and preventing mitochondrial oxidative stress, thus curtailing subsequent apoptotic events and cell death. ${ }^{18,31}$ Not only is melatonin itself a direct free radical scavenger but also metabolites that are formed during these interactions like N1-acetylN2-formyl-5-methoxykynuramine, which is deformylated to N1-acetyl-5-methoxykynuramine, and cyclic 3-hydroxymelatonin are also free radical scavengers. ${ }^{34}$ Thus, a cascade of metabolites of melatonin may contribute to the efficacy of the parent molecule to protect against oxidative stress. ${ }^{7}$ A major question to the view that antioxidants exert their healthprotective effects by 1-electron reactions with free radicals has been raised by Forman et al. ${ }^{35}$ By kinetic constraints, in vivo scavenging of radicals may be ineffective in antioxidant defense. Instead, enzymatic removal of nonradical electrophiles, such as hydroperoxides, in 2-electron redox reactions could be the major antioxidant mechanism. ${ }^{35}$ Indeed, the concept of radical avoidance was proposed to attempt to explain the protective effects of melatonin at the level of radical generation rather than detoxification of radicals already formed. ${ }^{36}$ If melatonin is capable of decreasing the processes leading to enhanced radical formation, this might be achieved by low concentrations of the methoxyindole. The isoforms of NAD(P)H oxidases (Nox) and the mitochondria should be mentioned as main sources of free radicals. Moreover, reactive 


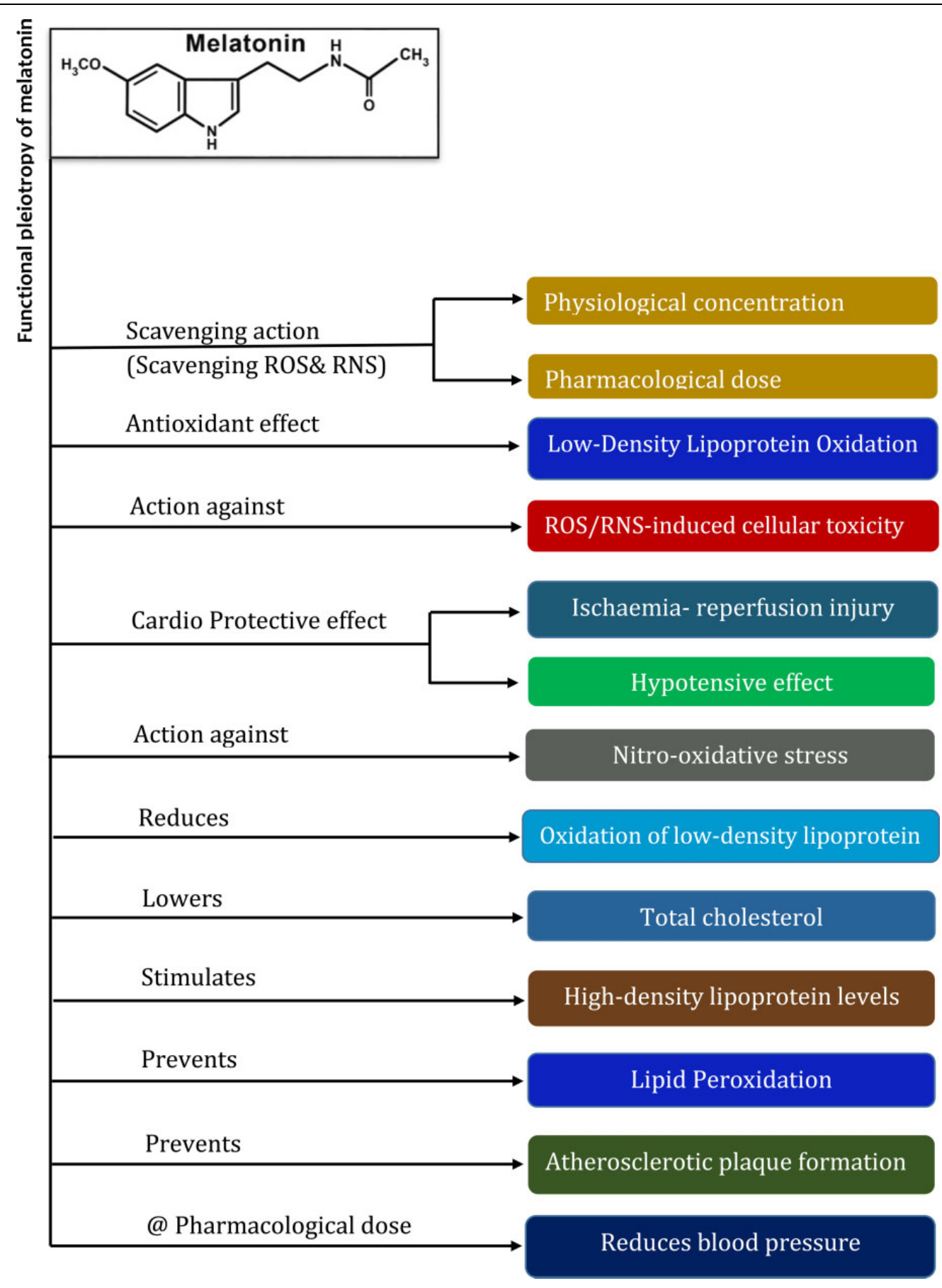

Figure I. Functional pleiotropy of melatonin.

nitrogen species can secondarily give rise to the formation of reactive oxygen species (ROS), both in and outside mitochondria, so that levels of oxidants can be considerably decreased by limitation of NO formation. Melatonin downregulates NO synthesis and inhibits ROS formation in microglia exposed to amyloid- $\beta$ 1-42 by preventing the phosphorylation of the p47 Nox subunit. ${ }^{37}$ Melatonin is also very effective to attenuate mitochondrial-free radical formation. Therefore, radical avoidance by melatonin must be recognized as a highly complex phenomenon, which comprises the integrative, orchestrating role of this molecule with its numerous actions at different levels. It should be noted that concentrations of melatonin may be sufficient for relevant direct scavenging in melatoninsynthesizing organs, especially pineal gland and Harderian gland. Whether accumulation in mitochondria leads to effective concentrations may be debated but is uncertain.

Melatonin displays a significant anti-inflammatory action and reduces the serum levels of oxidized low-density 
Table I. Reduction of Melatonin Secretion in Patients with CVD.

\begin{tabular}{lcc}
\hline Study Design & Population Characteristics, Sample Size & Melatonin Measurement \\
\hline Observational & I5 patients with coronary heart disease & Serum melatonin concentrations \\
cross- & (CHD), I0 healthy controls & were measured by \\
sectional & & radioimmunoassay at night \\
study & & $(02: 00$ hours) and afternoon \\
& & $(14: 00$ hours $)$
\end{tabular}

Observationa crosssectional study

48 male patients with severe CHD, 24 of 6-sulfatoxymelatonin (aMT6s) was them were taking $\beta$-blockers daily in therapeutic dosages. 18 age-matched healthy men served as controls (RIA) in overnight urine

Observational crosssectional study
Nocturnal excretion of aMT6s in urine collected from 18:00 to 06:00 hours. aMT6s was measured by a specific RIA
Observational crosssectional study
33 hospitalized patients with congestive heart failure (CHF) versus I 46 healthy ambulatory controls
Nocturnal excretion of aMT6s in urine collected from 18:00 to 06:00 hours. aMT6s was measured by a specific RIA

Melatonin was significantly lower in the 16 patients with CHD than in healthy controls (median 7.8 [interquartile range 6.5-II.8] vs 36.2 [32.2-42.5] pg/ $\mathrm{mL}, P<.000 \mathrm{I}$ ). Melatonin was undetectable in the afternoon

Night time urinary aMT6s levels were significantly lower in patients with CHD than in the control group $(F=16.8, P<.00 \mathrm{I}) \beta$-adrenoceptor blocker treatment had no significant influence in these patients $(F=0.052)$

Urinary aMT6s was significantly lower in patients with unstable angina than in healthy participants or in patients with stable angina. It correlated negatively with age in healthy participants, but not in patients with coronary disease. aMT6s in patients treated with $\beta$-adrenoceptor blockers did not differ significantly from patients with coronary disease not receiving $\beta$-blockers.

aMT6s levels were lower in patients with CHF than controls (median $2.6 \mu \mathrm{g}$ vs $6.02 \mu \mathrm{g}, P<.000 \mathrm{I}$ ). This decrease was observed regardless of $\beta$-adrenergic blocker treatment. There were no significant differences in urinary aMT6s levels between patients with chronic and acute CHF. A significant decrease in aMT6s excretion occurred with age

Observational 16 elderly patients with essential crosssectional study either dippers (DIP, $\mathrm{n}=8$ ) or hypertension, patients were defined as nondippers (NDIP, $\mathrm{n}=8$ ) according to the nocturnal change in the mean arterial pressure

\section{Observational 16 patients with angiographically cross- sectional} study
aMT6s was determined by ELISA in 2 separate urine collections, $I$ in the daytime and I during the night

Blood samples were collected every 2 hours between 22:00 and 08:00 hours. Melatonin levels were measured by RIA
Observational crosssectional study
190 patients with primary hypertension exhibiting a dipping and nondipping BP profile ( 88 men and 102 women)
Plasma melatonin was measured at the middle of the daytime and nighttime by RIA in DIP (3.28 $\pm 0.87 \mu \mathrm{g} / \mathrm{I} 2$ hours) and NDIP $(2.31 \pm 0.68 \mu \mathrm{g} / \mathrm{I} 2$ hours; $P=$ .39). Although DIP presented the physiological nocturnal increase in urinary aMT6s $(8.19 \pm 1.68 \mu \mathrm{g} / \mathrm{I} 2$ hours), this surge of melatonin production was missing in NDIP

Patients with CAD secreted less nocturnal melatonin at 02:00, 04:00, and 08:00 hours than control participants $(P=.014, P=.04$, and $P=.025$, respectively). Peak and delta melatonin (peak-lowest melatonin) were significantly lower in patients with CAD $(P=.006$ and $P=.002$, respectively). Peak time of melatonin secretion was observed earlier in patients with CAD

When patients were divided into dippers and nondippers on the basis of mean arterial or diastolic BP, a lower ratio of night/day melatonin concentration was found in nondippers than in dippers. There was a blunted night/day difference in plasma melatonin concentrations in patients with hypertension with the nondipping profile in diastolic BP 
Table I. (continued)

\begin{tabular}{|c|c|c|c|c|}
\hline Study Design & Population Characteristics, Sample Size & Melatonin Measurement & Results & Ref. \\
\hline $\begin{array}{l}\text { Observational } \\
\text { cross- } \\
\text { sectional } \\
\text { study }\end{array}$ & $\begin{array}{l}\text { I80 consecutive patients with a first } \\
\text { ST-segment elevation myocardial } \\
\text { infarction who underwent primary } \\
\text { percutaneous coronary intervention } \\
\text { within } 6 \text { hours from onset of } \\
\text { symptoms }\end{array}$ & $\begin{array}{l}\text { Intraplatelet melatonin levels were } \\
\text { measured in platelet-rich plasma } \\
\text { using an enzymatic immunoassay } \\
\text { procedure }\end{array}$ & $\begin{array}{l}\text { Patients with angiographic no-reflow had } \\
\text { lower intraplatelet melatonin levels } \\
\text { compared to patients without no- } \\
\text { reflow }(I 2.32 \pm 3.64 \text { vs } 18.62 \pm 3.88 \\
\text { ng/I00 } 000 \text { platelets, } P<.000 \mathrm{I}) \text {. After } \\
\text { adjusting by potential confounders, } \\
\text { binary logistic regression analysis } \\
\text { indicated that intraplatelet melatonin } \\
\text { levels were the only significant } \\
\text { predictor of angiographic no-reflow } \\
\text { (odds ratio I.58, } P<.000 \mathrm{I} \text { ) }\end{array}$ & 28 \\
\hline $\begin{array}{l}\text { Observational } \\
\text { longitudinal } \\
\text { study }\end{array}$ & $\begin{array}{l}554 \text { young women without baseline } \\
\text { hypertension }\end{array}$ & $\begin{array}{l}\text { First morning urine melatonin } \\
\text { levels }\end{array}$ & $\begin{array}{l}\text { During } 8 \text { years of follow-up, a total of } \\
\text { I } 25 \text { women developed hypertension. } \\
\text { The relative risk for incident } \\
\text { hypertension among women in the } \\
\text { highest quartile of urinary melatonin } \\
\text { ( }>27.0 \mathrm{ng} \text { per } \mathrm{mg} \text { creatinine) } \\
\text { compared to the lowest quartile } \\
(<10 . \mathrm{I} \mathrm{ng} \text { per mg creatinine) was } 0.49 \\
(95 \% \mathrm{Cl}, 0.28-0.85 ; P<.00 \mathrm{I})\end{array}$ & 29 \\
\hline
\end{tabular}

Abbreviations: BP, blood pressure; ELISA, enzyme-linked immunosorbent assay.

lipoprotein (LDL) responsible for atherogenic vascular formations. ${ }^{38,39}$ Indeed, oxidized LDL participates in the initiation and progression of atherosclerosis and contributes to endothelial dysfunction and plaque destabilization through multiple mechanisms. ${ }^{40}$ In vitro melatonin was found to inhibit oxidative LDL modification, ${ }^{41}$ a process that may translate in reduced formation of atherogenic plaques in vivo. Melatonin also decreases the formation of cholesterol and reduces LDL accumulation in freshly isolated human mononuclear leukocyte. ${ }^{42}$ However, not all studies have reported the LDLlowering effect of melatonin. ${ }^{43}$

Cyclophilin A is a ubiquitously expressed protein that has been highlighted as a major secreted oxidative stress-induced factor in atherosclerosis. In a study evaluating the role of cyclophilin A in the early phase of atherosclerosis, the atheroprotective effect of melatonin was assessed. ${ }^{44}$ Cyclophilin A expression increased and modulated inflammatory cell adhesion and interleukin 6 expression inducing vascular smooth muscle cell migration and inflammatory cell extravasation. All these effects were prevented by melatonin, indicating that melatonin treatment may represent a new atheroprotective approach that contributes to reducing the early phase of atherosclerosis. ${ }^{45}$

Melatonin inhibits several physiological processes in human platelets including the aggregation phenomenon, the release of ATP and serotonin (indexes of the platelet secretory mechanism), and the production of thromboxane B2. ${ }^{11,12}$

In an experimental study with an isolated perfused heart model in which the anterior descending coronary artery was temporarily ligated, infusion of melatonin $(1-250 \mathrm{mmol} / \mathrm{L})$ during the ischemic and reperfusion episodes prevented the occurrence of arrhythmias including premature ventricular contraction and ventricular fibrillation, which have been shown to occur on reperfusion without the infusion of melatonin. ${ }^{46}$ Protective effects of melatonin shortly after coronary artery ligation and in the absence of ischemia reperfusion were also reported. ${ }^{47}$ In a recent study using genetically engineered mice, it was demonstrated that nuclear melatonin receptor $\mathrm{ROR} \alpha$ may serve as an endogenous defender against ischemia reperfusion injury and may mediate the beneficial effect of melatonin on myocardial ischemia and reperfusion injury. ${ }^{48}$ Likewise, the ex vivo pretreatment with melatonin improved survival and function of adipose tissue-derived mesenchymal stem cells in vitro and in vivo, and by using a rat model of myocardial infarction, it was found that melatonin pretreatment enhanced the viability of engrafted stem cells and promoted their therapeutic potency. ${ }^{49}$ In view of these experimental and observational cross-sectional studies, melatonin might exert a cytoprotective effect at the level of human heart.

\section{Melatonin Levels in CVDs}

Individuals with elevated LDL/cholesterol levels have been reported to have low circulating levels of melatonin, ${ }^{50}$ and low melatonin levels have been reported in patients with CAD (Table 1). In an observational cross-sectional study of 15 individuals with CAD versus 10 healthy participants, melatonin was significantly lower in the patients with CAD than in the healthy controls. ${ }^{16}$ This is consistent with analysis of data from another observational cross-sectional study, which reported that nighttime urinary aMT6s levels were significantly lower in patients with CAD than in the control group. ${ }^{24}$ Significantly lower urinary aMT6s levels were reported in patients with unstable angina or in patients with stable angina. ${ }^{25}$ Yaprak 
et al reported that patients with CAD secreted less nocturnal melatonin at 02:00, 04:00, and 08:00 hours than controls.$^{26}$ In another related study of 180 consecutive patients with a first ST-segment elevation myocardial infarction who underwent percutaneous coronary intervention within 6 hours from onset of symptoms, patients with angiographic no-reflow had lower intraplatelet melatonin levels compared to patients without noreflow. ${ }^{28}$ Intraplatelet melatonin levels were the only significant predictor of angiographic no-reflow after adjusting for potential confounders.

Low urinary aMT6s excretion was reported in CHF, a decrease that was observed regardless of $\beta$-adrenergic blocker. There were no significant differences in the low urinary aMT6s levels between patients with chronic and acute CHF. ${ }^{15}$

Concerning patients with hypertension, there were reports indicating the suppression of nocturnal melatonin secretion in nondippers, ${ }^{14,27}$ and in an observational longitudinal study of 554 young women without baseline hypertension, the relative risk for incident hypertension among women in the highest quartile of urinary melatonin was about half that in the lowest quartile. $^{29}$

\section{Melatonin Effects on Arterial Blood Pressure in Humans}

Numerous pharmacological and nonpharmacological procedures have been used in the treatment of hypertension; however, the percentage of individuals with uncontrolled hypertension still remains unacceptably high. ${ }^{51}$

The effects of melatonin on cardiovascular function in healthy participants are significant (Table 2). Melatonin in comparison to placebo was able to reduce blood pressure (BP), vascular reactivity, the pulsatility index in the internal carotid artery, and circulating catecholamines in healthy participants. ${ }^{13,52,53}$ In another related study comparing postmenopausal women with and without hormone replacement therapy (HRT), melatonin reduced internal carotid artery pulsatility index, systolic and diastolic BP, and increased the NO level in HRT-treated women only, suggesting that several effects of melatonin may be modulated by gonadal steroids. ${ }^{54}$ As shown by power spectral analysis of heart rate variability and BP monitoring, melatonin administration increased cardiac vagal tone and reduced plasma norepinephrine and dopamine levels in the supine position in awake healthy volunteers. ${ }^{55}$ Although BP was reduced significantly, heart rate and burst rate of muscle sympathetic nerve activity (MSNA) did not change significantly after melatonin. ${ }^{56}$ However, in another study examining the sympathetic nerve responses to orthostatic stress, the increase in MSNA was smaller in the melatonin-treated group. ${ }^{57}$

Blunted decline in the physiological BP's nocturnal fall, the nondipper pattern, is associated with hypertension-induced organ damage such as left ventricular hypertrophy, microalbuminuria, reduced arterial compliance, and worse prognosis in terms of cardiovascular events. ${ }^{58}$ As shown in Table 3, melatonin treatment can be useful in this kind of patients.
A double-blind, placebo-controlled study demonstrated that melatonin given orally $(2.5 \mathrm{mg} / \mathrm{d})$ for 3 weeks to patients with essential hypertension significantly reduced both systolic and diastolic BP. ${ }^{60}$ Nondipper hypertensives have also been found to have a missing surge of melatonin production at nighttime compared to hypertensives who had an appropriate reduction in BP at nighttime (Table 1). ${ }^{14,27}$

In a meta-analysis performed on the effect of melatonin on nocturnal BP, the combination of controlled-release melatonin and antihypertensive treatment was found effective and safe in ameliorating nocturnal hypertension, whereas fast-release melatonin was not. ${ }^{61}$ The data differed from a former report indicating that the evening administration of melatonin induced an increase of BP and heart rate in patients with hypertension well controlled by nifedipine. ${ }^{59}$ These discrepancies underline the necessity of further studies on the matter.

It has been suggested that the reduction in nocturnal BP by repeated melatonin intake at night is attributable to its curing effect on the circadian output of the suprachiasmatic nucleus. ${ }^{64}$ The normalization of circadian pacemaker function in the regulation of BP by melatonin treatment has thus been proposed as a potential strategy for the treatment of essential hypertension.

The vasoregulatory actions of melatonin are complex and may involve both central and peripheral mechanisms. ${ }^{65,66}$ The responses elicited by the activation of MT1 (vasoconstriction) and MT2 (vasodilation) are dependent on circadian time, duration, and mode of exposure to endogenous or exogenous melatonin, as well as of functional receptor sensitivity.

\section{Potential Use of Melatonin in Pulmonary Hypertension}

Oxidative stress has been proposed as one of the major mechanisms leading to the development of pulmonary hypertension. ${ }^{67,68}$ Therefore, it is reasonable to explore the effect of antioxidant therapy in pulmonary hypertension. As discussed above, melatonin has a potent antioxidant activity, which can reduce antioxidant damage in cardiovascular tissues.

Three recent animal studies have suggested that melatonin may be beneficial in hypoxic pulmonary hypertension. In one study performed in newborn sheep gestated, born, and raised at $3600 \mathrm{~m}$, melatonin reduced pulmonary artery pressure and resistance for the first 3 days of treatment and significantly improved the vasodilator function of small pulmonary arteries, reduced pulmonary oxidative stress markers, and increased enzymatic and nonenzymatic antioxidant capacity. ${ }^{69}$

In another study performed in Sprague Dawley rats exposed to intermittent chronic hypoxia for 4 weeks to induce hypoxic pulmonary hypertension, ${ }^{70}$ melatonin administration attenuated the elevation of right ventricular pressure and reduced the pulmonary vascular structure remodeling. In line with these findings, a third study assessed the effect of melatonin as a curative or preventive therapy of pulmonary hypertension in Long Evans rats in which pulmonary hypertension had been induced by injecting monocrotaline. Melatonin was administered 5 days prior to or 14 days after the injection of 
Table 2. Effects of Melatonin on Cardiovascular Function in Healthy Humans.

\begin{tabular}{lll}
\hline Study Design & Population & Experiment \\
\hline $\begin{array}{l}\text { Placebo-controlled } \\
\text { study }\end{array}$ & 12 young women & Melatonin I mg oral tablet between \\
& & $14: 30$ and $17: 30$ hours, 90 minutes \\
& before investigation
\end{tabular}

Placebo-controlled 17 young, healthy, early follicularstudy

Randomized, double-blind, placebocontrolled study
Randomized, double-blind placebocontrolled study
23 postmenopausal women of which 12 were on the estrogenic phase and II were under hormone replacement therapy (HRT) and with continuous transdermal estradiol plus cyclic medroxyprogesterone acetate
Placebo-controlled 26 healthy men study

Observational
study

Placebo-controlled II healthy participants study
Melatonin I mg oral tablet between I 4:30 and I7:30 hours, 90 minutes before investigation

Melatonin I mg oral tablet between 14:30 and 17:30 hours, 90 minutes before investigation
Results

Ref.

The administration of melatonin

significantly reduced $\mathrm{BP}$, the pulsatility index in the internal carotid artery, and catecholamines levels

The administration of melatonin significantly reduced $\mathrm{BP}$, the pulsatility index in the internal carotid artery, and catecholamines levels

The administration of melatonin significantly reduced $\mathrm{BP}$, the pulsatility index in the internal carotid artery, and catecholamines levels. The effect of melatonin on pulsatility index was related to baseline values, being greater in men with higher baseline values

In untreated postmenopausal women, melatonin treatment was ineffective, while in HRT-treated women, studied during the estrogenic phase, melatonin reduced, within 90 minutes, systolic $(-8.1 \pm 9.9 \mathrm{~mm} \mathrm{Hg}$; $P=.054)$, diastolic $(-5.0 \pm 7.0$ $\mathrm{mm} \mathrm{Hg} ; P=.049)$, and mean $(-6.0 \pm 6.6 \mathrm{~mm} \mathrm{Hg} ; P=.037)$ BP. Norepinephrine but not epinephrine levels were also reduced. Similarly, resistance to blood flow in the internal carotid artery was decreased by melatonin

Compared with placebo, melatonin 55 administration increased R-R interval, the square root of the mean of the squared differences between adjacent normal R-R intervals, melatonin decreased the low-frequency to high-frequency ratio and $\mathrm{BP}$ in the supine position $(P<.01)$. Plasma norepinephrine and dopamine levels in the supine position after melatonin administration were significantly lower than in placebo

$3 \mathrm{mg}$ of melatonin was given and the $\mathrm{BP}$, heart rate, and muscle sympathetic nerve activity (MSNA) were recorded continuously for 80 minutes

50 minutes after receiving a $3 \mathrm{mg}$ tablet of melatonin or placebo (in different days) sympathetic nerve responses to orthostatic stress (MSNA), arterial BP, heart rate, forearm blood flow, and thoracic impedance were measured
$B P$ was reduced significantly, while heart rate and burst rate of MSNA did not change significantly

During the placebo trial, MSNA increased by $33 \% \pm 8 \%$ and $251 \% \pm$ $70 \%$ during -10 and $-40 \mathrm{~mm} \mathrm{Hg}$, respectively, but increased by only $8 \% \pm 7 \%$ and $111 \% \pm 35 \%$ during -10 and $-40 \mathrm{~mm} \mathrm{Hg}$ with melatonin, respectively $(P<.0 \mathrm{I})$. Arterial $\mathrm{BP}$ and forearm vascular resistance responses to orthostatic stress were unchanged by melatonin 
Table 3. Effect of Melatonin in Patients with Hypertension.

\begin{tabular}{lll}
\hline Study Design & Population & Experiment \\
\hline $\begin{array}{l}\text { Double-blind } \\
\text { crossover }\end{array}$ & 47 outpatients with mild-to-moderate & Melatonin $5 \mathrm{mg}$ at 22:30 hours. A \\
study & essential hypertension taking & 24-hour noninvasive ambulatory \\
& nifedipine 30 or $60 \mathrm{mg}$ as a & blood pressure (BP) monitoring \\
& monotherapy at $08: 30$ hours for at & was recorded from each patient \\
& least 3 months &
\end{tabular}

$\begin{gathered}\text { Double-blind, } \\ \text { placebo- }\end{gathered}$
controlled,
crossover with untreated essential
study

Randomized, double-blind, placebocontrolled study

Combined analysis of controlled clinical trials
38 patients with treated hypertension (22 males) with confirmed nocturnal hypertension (mean nighttime systolic BP > I $25 \mathrm{~mm} \mathrm{Hg}$ ), according to repeated 24-hour ambulatory blood pressure monitoring

\section{Post hoc analysis of pooled antihypertensive drug-treated subpopulations from 4 randomized, double-blind trials}

$2.5 \mathrm{mg}$ of oral melatonin given as a single and repeated (daily for 3 weeks) dose I hour before sleep. 24-hour ambulatory blood pressure and actigraphic estimates of sleep quality

Results

Ref.

The evening administration of melatonin increased BP and heart rate (HR) throughout the 24-hour period (Delta SBP $=+6.5 \mathrm{~mm} \mathrm{Hg}$, $P<.001$; Delta DBP $=+4.9 \mathrm{~mm}$ $\mathrm{Hg}, P<.0 \mathrm{I}$; Delta HR $=+3.9$ beats/minute, $P<.0 \mathrm{I}$ ). The increase in DBP as well as HR was particularly evident during the morning and the afternoon hours

Repeated melatonin intake reduced systolic and diastolic blood pressure during sleep by 6 and 4 $\mathrm{mm} \mathrm{Hg}$, respectively. Heart rate was not affected. A single dose of melatonin had no effect on blood pressure. Repeated doses of melatonin also improved sleep $P<.05$

Controlled-release melatonin $2 \mathrm{mg}$ or placebo 2 hours before bedtime for 4 weeks. 24-hour ambulatory blood pressure monitoring

Melatonin treatment reduced nocturnal systolic BP significantly from $136 \pm 9$ to $130 \pm 10 \mathrm{~mm} \mathrm{Hg}$ $(P=.0 \mathrm{II})$, and diastolic BP from 72 $\pm \mathrm{II}$ to $69 \pm 9 \mathrm{~mm} \mathrm{Hg}(P=.002)$, whereas placebo had no effect on nocturnal BP. The reduction in nocturnal systolic BP was significantly greater with melatonin than with placebo $(P=.0 \mathrm{I})$, and was most prominent between 2:00 AM and 5:00 AM $(P=.002)$

Prolonged-release melatonin (PRM; 2 Compared to placebo, PRM had $\mathrm{mg}$ ) for 3 weeks or 28 weeks. Measured the efficacy (by Leeds Sleep Evaluation Questionnaire scores of quality of sleep and alertness and behavioral integrity, sleep latency, and Clinical Global Impression of Improvement) and safety of PRM for primary insomnia in patients aged 55 years and older who were treated with antihypertensive drugs significantly improved quality of sleep and behavior following awakening $(P<.0008)$. Sleep latency $(P=.02)$ and $C G I-I$ $(P=.0003)$ also improved significantly. No differences were observed between PRM and placebo groups in daytime blood pressure at baseline and treatment phases. The rate of adverse events normalized per 100 patient-weeks was lower for PRM (3.66) than for placebo (8.53)

Randomized, 16 patients with hypertension double-blind, (age 45-64 years; 9 women) treated with the $\beta$-blockers atenolol or placebocontrolled, parallel-group metoprolol

Melatonin $2.5 \mathrm{mg}$ nightly for 3 weeks. Sleep quality measured by polysomnography

Melatonin supplementation for 3 weeks significantly increased total sleep time $(+36$ minutes; $P=.046)$, increased sleep efficiency $(+7.6 \%$; $P=.046)$, decreased sleep onset latency to Stage 2 (- 14 minutes; $P=.00 \mathrm{I})$, increased Stage 2 sleep $(+4 I$ minutes; $P=.037)$ when compared to placebo. The sleep onset latency remained significantly shortened on the night after discontinuation of melatonin administration ( -25 minutes; $P=.00 \mathrm{I}$ ), suggesting a carry-over effect 
monocrotaline. The study showed that both curative and preventive treatment improved right ventricular functional and plasma oxidative stress parameters and reduced cardiac interstitial fibrosis. ${ }^{71}$

Therefore, melatonin seems to confer beneficial effects in pulmonary hypertension via antioxidant, anti-inflammatory, and antiproliferative mechanisms. Clinical investigation of the effects of melatonin on right ventricle hemodynamic function in patients with pulmonary hypertension is warranted.

\section{Melatonin Dose and Safety}

The majority of clinical trials on the therapeutic usefulness of melatonin in different fields of medicine have shown very low toxicity of melatonin over a wide range of doses. ${ }^{72}$ Doses of melatonin that considerably exceed those used in cardiovascular disorders have been found to be safe. In the treatment of amyotrophic lateral sclerosis, patients received either $60 \mathrm{mg} / \mathrm{d}$ orally for up to 13 months $^{73}$ or enteral doses of $300 \mathrm{mg} / \mathrm{d}$ for up to 2 years. ${ }^{74}$ In a phase 1 dose escalation study in healthy volunteers to assess the tolerability and pharmacokinetics of $20,30,50$, and $100 \mathrm{mg}$ oral doses of melatonin, no adverse effects after oral melatonin, other than mild transient drowsiness with no effects on sleeping patterns, were seen. ${ }^{75}$ Therefore, further clinical trials using dosages of melatonin in the range of 50 to $100 \mathrm{mg} / \mathrm{d}$ appear to be reasonable and are warranted. The priorities for populations, outcomes, and durations of these studies must be defined.

\section{Conclusion}

The possible therapeutic role of melatonin in CVDs is increasingly apparent, especially with potential benefits in the reduction of ischemia-reperfusion injury and decreasing nocturnal BP. The data suggest that preserving endogenous melatonin levels, or the use of melatonin supplements, may be beneficial in CVDs.

Melatonin is available in pharmacologically pure form, is relatively inexpensive, is absorbed when administered via any route, and its toxicity is remarkably low. Considering that CVDs are the leading cause of death globally, ${ }^{1}$ the fact that melatonin has been found to be cardioprotective and possess low toxicity could have important clinical implications. Therefore, more extensive, large-size clinical trials are needed to evaluate melatonin's efficacy as a novel therapeutic intervention in CVDs.

\section{Author Contributions}

Seithikurippu R. Pandi-Perumal, Ahmed S. BaHammam, Gregory M. Brown, and Daniel P. Cardinali contributed to conception, drafted the manuscript, critically revised the manuscript, gave final approval, and agree to be accountable for all aspects of work ensuring integrity and accuracy. Nwakile I. Ojike, Oluwaseun A. Akinseye, Tetyana Kendzerska, Kenneth Buttoo, and Perundurai S. Dhandapany drafted the manuscript, critically revised the manuscript, gave final approval, and agree to be accountable for all aspects of work ensuring integrity and accuracy.

\section{Declaration of Conflicting Interests}

The author(s) declared no potential conflicts of interest with respect to the research, authorship, and/or publication of this article.

\section{Funding}

The author(s) disclosed receipt of the following financial support for the research, authorship, and/or publication of this article: This work was supported by a grant from the Agencia Nacional de Promoción Científica y Tecnológica, Argentina (PICT 2012, 0984).

\section{References}

1. World Health Organization. Cardiovascular diseases (CVDs). 2016. Web site. http://www.who.int/mediacentre/factsheets/fs3 17/en/. Accessed June 6, 2016.

2. Mozaffarian D, Benjamin EJ, GO AS, et al. Heart Disease and Stroke Statistics-2016 Update: A Report From the American Heart Association. Circulation. Jan 26 2016;133(4):e38-60.

3. US Department of Health Human Services. A public health action plan to prevent heart disease and stroke. Atlanta, GA: US Department of Health and Human Services, Centers for Disease Control and Prevention 2003. Web site. https:// www.cdc.gov/dhdsp/action_plan/pdfs/action_plan_2of7.pdf. Accessed June 6, 2016.

4. Minneman KP, Wurtman RJ. The pharmacology of the pineal gland. Annu Rev Pharmacol Toxicol. 1976;16:33-51.

5. Dominguez-Rodriguez A, Abreu-Gonzalez P, Reiter RJ. Melatonin and cardiovascular disease: myth or reality? Revista Española De Cardiología (English Edition). 2012;65(3):215-218.

6. Ekmekcioglu C, Thalhammer T, Humpeler S, et al. The melatonin receptor subtype MT2 is present in the human cardiovascular system. J Pineal Res. 2003;35(1):40-44.

7. Hardeland R, Cardinali DP, Srinivasan V, Spence DW, Brown GM, Pandi-Perumal SR. Melatonin - a pleiotropic, orchestrating regulator molecule. Prog Neurobiol. 2011;93(3):350-384.

8. Lew MJ, Flanders S. Mechanisms of melatonin-induced vasoconstriction in the rat tail artery: a paradigm of weak vasoconstriction. Br J Pharmacol. 1999;126(6):1408-1418.

9. Liu J, Clough SJ, Hutchinson AJ, et al. MT1 and MT2 melatonin receptors: a therapeutic perspective. Ann Rev Pharmacol Toxicol. 2016;56:361-383.

10. Grossman E, Laudon M, Zisapel N. Effect of melatonin on nocturnal blood pressure: meta-analysis of randomized controlled trials. Vasc Health Risk Manag. 2011;7:577-584.

11. Del Zar MM, Martinuzzo M, Falcón C, Cardinali DP, Carreras LO, Vacas MI. Inhibition of human platelet aggregation and thromboxane-B2 production by melatonin: evidence for a diurnal variation. J Clin Endocrinol Metab. 1990;70(1):246-251.

12. Del Zar MM, Martinuzzo M, Cardinali DP, Carreras LO, Vacas MI. Diurnal variation in melatonin effect on adenosine triphosphate and serotonin release by human platelets. Acta Endocrinol (Copenh). 1990;123(4):453-458.

13. Arangino S, Cagnacci A, Angiolucci M, et al. Effects of melatonin on vascular reactivity, catecholamine levels, and blood pressure in healthy men. Am J Cardiol. 1999;83(9):1417-1419. 
14. Jonas M, Garfinkel D, Zisapel N, Laudon M, Grossman E. Impaired nocturnal melatonin secretion in non-dipper hypertensive patients. Blood Press. 2003;12(1):19-24.

15. Girotti L, Lago M, Ianovsky O, et al. Low urinary 6sulfatoxymelatonin levels in patients with severe congestive heart failure. Endocrine. 2003;22(3):245-248.

16. Brugger P, Marktl W, Herold M. Impaired nocturnal secretion of melatonin in coronary heart disease. Lancet. 1995;345(8962): 1408.

17. Dominguez-Rodriguez A, Abreu-Gonzalez P, Garcia MJ, Sanchez J, Marrero F, de Armas-Trujillo D. Decreased nocturnal melatonin levels during acute myocardial infarction. J Pineal Res. 2002;33(4):248-252.

18. Acuña-Castroviejo D, Escames G, Venegas C, et al. Extrapineal melatonin: sources, regulation, and potential functions. Cell $\mathrm{Mol}$ Life Sci. 2014;71(16):2997-3025.

19. Costa EJ, Lopes RH, Lamy-Freund MT. Permeability of pure lipid bilayers to melatonin. J Pineal Res. 1995;19(3):123-126.

20. Venegas C, Garcia JA, Escames G, et al. Extrapineal melatonin: analysis of its subcellular distribution and daily fluctuations. $J$ Pineal Res. 2012;52(2):217-227.

21. Ekmekcioglu C. Melatonin receptors in humans: biological role and clinical relevance. Biomed Pharmacother. 2006;60(3): 97-108.

22. Bojkowski CJ, Arendt J. Factors influencing urinary 6sulphatoxymelatonin, a major melatonin metabolite, in normal human subjects. Clin Endocrinol. 1990;33(4):435-444.

23. Bubenik GA, Pang SF. The role of serotonin and melatonin in gastrointestinal physiology: ontogeny, regulation of food intake, and mutual serotonin-melatonin feedback. J Pineal Res. 1994; 16(2):91-99.

24. Sakotnik A, Liebmann PM, Stoschitzky K, et al. Decreased melatonin synthesis in patients with coronary artery disease. Eur Heart Journal. 1999;20(18):1314-1317.

25. Girotti L, Lago M, Ianovsky O, et al. Low urinary 6sulphatoxymelatonin levels in patients with coronary artery disease. J Pineal Res. 2000;29(3):138-142.

26. Yaprak M, Altun A, Vardar A, Aktoz M, Ciftci S, Ozbay G. Decreased nocturnal synthesis of melatonin in patients with coronary artery disease. Int J Cardiol. 2003;89(1):103-107.

27. Zeman M, Dulkova K, Bada V, Herichova I. Plasma melatonin concentrations in hypertensive patients with the dipping and non-dipping blood pressure profile. Life Sci. 2005;76(16): 1795-1803.

28. Dominguez-Rodriguez A, Abreu-Gonzalez P, Jimenez-Sosa A, Avanzas P, Bosa-Ojeda F, Kaski JC. Usefulness of intraplatelet melatonin levels to predict angiographic no-reflow after primary percutaneous coronary intervention in patients with ST-segment elevation myocardial infarction. Am J Cardiol. 2010;106(11): 1540-1544.

29. Forman JP, Curhan GC, Schernhammer ES. Urinary melatonin and risk of incident hypertension among young women. J Hypertens. 2010;28(3):446-451.

30. Reiter RJ, Tan DX. Melatonin: a novel protective agent against oxidative injury of the ischemic/reperfused heart. Cardiovasc Res. 2003;58(1):10-19.
31. Leon J, Acuna-Castroviejo D, Escames G, Tan DX, Reiter RJ. Melatonin mitigates mitochondrial malfunction. J Pineal Res. 2005;38(1):1-9.

32. Akbulut KG, Akbulut H, Akgun N, Gonul B. Melatonin decreases apoptosis in gastric mucosa during aging. Aging Clin Exp Res. 2012;24(1):15-20.

33. Tajes Orduna M, Pelegri Gabalda C, Vilaplana Hortensi J, Pallas Lliberia M, Camins Espuny A. An evaluation of the neuroprotective effects of melatonin in an in vitro experimental model of age-induced neuronal apoptosis. J Pineal Res. 2009;46(3): 262-267.

34. Manda K, Ueno M, Anzai K. AFMK, a melatonin metabolite, attenuates X-ray-induced oxidative damage to DNA, proteins and lipids in mice. J Pineal Res. 2007;42(4):386-393.

35. Forman HJ, Davies KJ, Ursini F. How do nutritional antioxidants really work: nucleophilic tone and para-hormesis versus free radical scavenging in vivo. Free Radic Biol Med. 2014;66: 24-35.

36. Hardeland R. Antioxidative protection by melatonin: multiplicity of mechanisms from radical detoxification to radical avoidance. Endocrine. 2005;27(2):119-130.

37. Zhou J, Zhang S, Zhao X, Wei T. Melatonin impairs NADPH oxidase assembly and decreases superoxide anion production in microglia exposed to amyloid-beta1-42. J Pineal Res. 2008;45(2): 157-165.

38. Cuzzocrea S, Thiemermann C, Salvemini D. Potential therapeutic effect of antioxidant therapy in shock and inflammation. Curr Med Chem. 2004;11(9):1147-1162.

39. Hardeland R, Cardinali DP, Brown GM, Pandi-Perumal SR. Melatonin and brain inflammaging. Prog Neurobiol. 2015;127-128: 46-63.

40. Landmesser U, Harrison DG. Oxidant stress as a marker for cardiovascular events: Ox marks the spot. Circulation. 2001; 104(22):2638-2640.

41. Kelly MR, Loo G. Melatonin inhibits oxidative modification of human low-density lipoprotein. J Pineal Res. 1997;22(4): 203-209.

42. Mullerwieland D, Behnke B, Koopmann K, Krone W. Melatonin inhibits LDL receptor activity and cholesterol-synthesis in freshly isolated human mononuclear leukocytes. Biochem Biophys Res Commun. 1994;203(1):416-421.

43. Wakatsuki A, Okatani Y, Ikenoue N, Kaneda C, Fukaya T. Effects of short-term melatonin administration on lipoprotein metabolism in normolipidemic postmenopausal women. Maturitas. 2001; 38(2):171-177.

44. Rezzani R, Favero G, Stacchiotti A, Rodella LF. Endothelial and vascular smooth muscle cell dysfunction mediated by cyclophylin A and the atheroprotective effects of melatonin. Life Sci. 2013; 92(17-19):875-882.

45. Favero G, Rodella LF, Reiter RJ, Rezzani R. Melatonin and its atheroprotective effects: a review. Mol Cell Endocrinol. 2014; 382(2):926-937.

46. Tan DX, Manchester LC, Reiter RJ, Qi W, Kim SJ, El-Sokkary GH. Ischemia/reperfusion-induced arrhythmias in the isolated rat heart: prevention by melatonin. J Pineal Res. 1998;25(3): 184-191. 
47. Castagnino HE, Lago N, Centrella JM, et al. Cytoprotection by melatonin and growth hormone in early rat myocardial infarction as revealed by Feulgen DNA staining. Neuroendocrinol Lett. 2002;23(5-6):391-395.

48. He B, Zhao Y, Xu L, et al. The nuclear melatonin receptor RORalpha is a novel endogenous defender against myocardial ischemia/reperfusion injury. J Pineal Res. 2016;60(3):313-326.

49. Zhu P, Liu J, Shi J, et al. Melatonin protects ADSCs from ROS and enhances their therapeutic potency in a rat model of myocardial infarction. J Cell Mol Med. 2015;19(9):2232-2243.

50. Dominguez-Rodriguez A, Abreu-Gonzalez P, Sanchez-Sanchez JJ, Kaski JC, Reiter RJ. Melatonin and circadian biology in human cardiovascular disease. J Pineal Res. 2010;49(1):14-22.

51. Akinseye OA, Akinseye LI. Home blood pressure monitoring and hypertension control. Primary Health Care. 2015;5(182): 2167-1079.1000182.

52. Cagnacci A, Arangino S, Angiolucci M, Maschio E, Longu G, Metis GB. Potentially beneficial cardiovascular effects of melatonin administration in women. J Pineal Res. 1997;22(1):16-19.

53. Cagnacci A, Arangino S, Angiolucci M, Maschio E, Melis GB. Influences of melatonin administration on the circulation of women. Am J Physiol. 1998;274(2 pt 2):R335-R338.

54. Cagnacci A, Arangino S, Angiolucci M, et al. Effect of exogenous melatonin on vascular reactivity and nitric oxide in postmenopausal women: role of hormone replacement therapy. Clin Endocrinol. 2001;54(2):261-266.

55. Nishiyama K, Yasue H, Moriyama Y, et al. Acute effects of melatonin administration on cardiovascular autonomic regulation in healthy men. Am Heart J. 2001;141(5):13A-17A.

56. Kitajima T, Kanbayashi T, Saitoh Y, et al. The effects of oral melatonin on the autonomic function in healthy subjects. Psychiatry Clin Neurosci. 2001;55(3):299-300.

57. Ray CA. Melatonin attenuates the sympathetic nerve responses to orthostatic stress in humans. J Physiol. 2003;551(3): 1043-1048.

58. Redon J, Lurbe E. Nocturnal blood pressure versus nondipping pattern: what do they mean? Hypertension. 2008;51(1):41-42.

59. Lusardi P, Piazza E, Fogari R. Cardiovascular effects of melatonin in hypertensive patients well controlled by nifedipine: a $24-$ hour study. Br J Clin Pharmacol. 2000;49(5):423-427.

60. Scheer FA, Van Montfrans GA, van Someren EJ, Mairuhu G, Buijs RM. Daily nighttime melatonin reduces blood pressure in male patients with essential hypertension. Hypertension. 2004; 43(2):192-197.

61. Grossman E, Laudon M, Yalcin R, et al. Melatonin reduces night blood pressure in patients with nocturnal hypertension. Am J Med. 2006;119(10):898-902.
62. Lemoine P, Wade AG, Katz A, Nir T, Zisapel N. Efficacy and safety of prolonged-release melatonin for insomnia in middleaged and elderly patients with hypertension: a combined analysis of controlled clinical trials. Integr Blood Press Control. 2012;5: 9-17.

63. Scheer FA, Morris CJ, Garcia JI, et al. Repeated melatonin supplementation improves sleep in hypertensive patients treated with beta-blockers: a randomized controlled trial. Sleep. 2012;35(10): 1395-1402.

64. Scheer FA. Potential use of melatonin as adjunct antihypertensive therapy. Am J Hypertens. 2005;18(12 pt 1):1619-1620.

65. Dubocovich ML, Markowska M. Functional MT1 and MT2 melatonin receptors in mammals. Endocrine. 2005;27(2): 101-110

66. Xia CM, Shao CH, Xin L, et al. Effects of melatonin on blood pressure in stress-induced hypertension in rats. Clin Exp Pharmacol Physiol. 2008;35(10):1258-1264.

67. Masri FA, Comhair SAA, Dostanic-Larson I, et al. Deficiency of lung antioxidants in idiopathic pulmonary arterial hypertension. Clin Trans Sci. 2008;1(2):99-106.

68. Jane-Wit D, Chun HJ. Mechanisms of dysfunction in senescent pulmonary endothelium. J Gerontol A Biol Sci Med Sci. 2012; 67(3):236-241.

69. Torres F, González-Candia A, Montt C, et al. Melatonin reduces oxidative stress and improves vascular function in pulmonary hypertensive newborn sheep. J Pineal Res. 2015;58(3):362-373.

70. Jin H, Wang Y, Zhou L, et al. Melatonin attenuates hypoxic pulmonary hypertension by inhibiting the inflammation and the proliferation of pulmonary arterial smooth muscle cells. J Pineal Res. 2014;57(4):442-450.

71. Maarman G, Blackhurst D, Thienemann F, et al. Melatonin as a preventive and curative therapy against pulmonary hypertension. J Pineal Res. 2015;59(3):343-353.

72. Sanchez-Barcelo EJ, Mediavilla MD, Tan DX, Reiter RJ. Clinical uses of melatonin: evaluation of human trials. Curr Med Chem. 2010;17(19):2070-2095.

73. Jacob S, Poeggeler B, Weishaupt JH, et al. Melatonin as a candidate compound for neuroprotection in amyotrophic lateral sclerosis (ALS): high tolerability of daily oral melatonin administration in ALS patients. J Pineal Res. 2002;33(3):186-187.

74. Weishaupt JH, Bartels C, Polking E, et al. Reduced oxidative damage in ALS by high-dose enteral melatonin treatment. $J$ Pineal Res. 2006;41(4):313-323.

75. Galley HF, Lowes DA, Allen L, Cameron G, Aucott LS, Webster NR. Melatonin as a potential therapy for sepsis: a phase I dose escalation study and an ex vivo whole blood model under conditions of sepsis. J Pineal Res. 2014;56(4):427-438. 